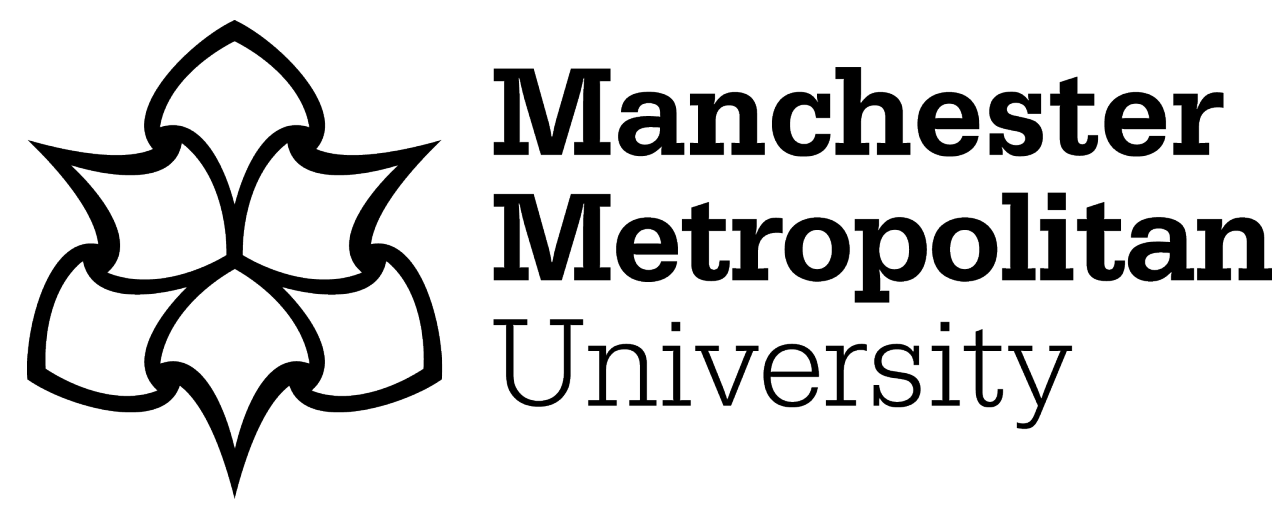

Greenhalgh, S, Finucane, L, Mercer, C and Selfe, James (2018) Assessment and management of cauda equina syndrome. Musculoskeletal Science and Practice, 37. pp. 69-74. ISSN 2468-7812

Downloaded from: https://e-space.mmu.ac.uk/621393/

Version: Accepted Version

Publisher: Elsevier

DOI: https://doi.org/10.1016/j.msksp.2018.06.002

Please cite the published version 


\title{
Assessment and Management of Cauda Equina Syndrome
}

\author{
Abstract \\ Introduction \\ Cauda equina syndrome (CES) is a rare condition that affects the nerves in the spine supplying the \\ bladder, bowel and sexual function. Identification and subsequent urgent action is required to \\ avoid permanent damage to these essential organs. Delays in diagnosis can have devastating and life \\ changing consequences for patients and result in high cost negligence claims.
}

Purpose

The purpose of this paper is to examine the current evidence and provide a consistent approach in the safe management of patients presenting with CES. It will include a focus on the importance of communication, documentation and a practical approach to safety netting those at risk.

Implications for practice

CES has significant implications for patients and clinicians alike. Timely, effective diagnosis and management of patients with CES results in a better outcome.

Key words; Cauda Equina Syndrome, Red Flags, Masqueraders, Safety Netting

\section{Introduction}

Cauda Equina Syndrome (CES) is a challenging condition to diagnose and manage. It may present at any time or in any setting and it is imperative that clinicians are able to quickly reason through their findings to manage the patient effectively. The purpose of this paper is to highlight the many challenges faced by clinicians in recognising and managing the condition and will offer guidance on the evidence based management of these patients.

There are many causes of CES, but the most common cause is that of a lumbar spine disc herniation and occurs most frequently between the ages of 31-50 (Fuso et al, 2013). Cauda equina compression usually occurs as a result of a disc prolapse, often at the $L 4 / 5$ level (Fraser et al, 2009). However, any space-occupying lesion, such as spinal stenosis, tumour, cysts, infection or bony ingress can narrow the spinal canal and cause compression of the cauda equina. So what is the scale of the problem? Most literature agrees that within the total back pain population CES is a rare condition. In 1994, The Clinical Standards Advisory Group suggested that serious pathology comprised just $1 \%$ of all back pain, with CES being just one of the many serious conditions that can lead to back pain (CSAG 1994, Verhagen et al, 2016). Despite CES being estimated to have a very low prevalence of just 0.04 (Jarvik, 2002), it is considered to be a major problem internationally and multiple National Guidelines for the management of low back pain refer to the importance of screening for CES (Verhagen et al 2016). It is important to note that whilst the published evidence is that in the general population CES is rare, in an eighteen-month period in a Primary Care Interface service in the UK, 28 positive CES patients were managed as emergency cases and referred to a specialist spinal 
service, representing 3.5\% of the service's patient population. In 1995 the Office of National Statistics estimated that in the UK one CES patient will present annually for every 50,000 patients seen in primary care, the equivalent of $0.002 \%$. These varying statistics appear to be contradictory, however, they can be explained by understanding the clinical context in which these patients were seen. Underwood (2009) states that a GP will see one CES patient in their entire career yet Fairbank (2014) identifies that 1000 operations are carried out per year in the UK for CES. The incidence of CES patients attending a particular medical setting depends on the type of the medical service provided. Spinal surgeons will always see more CES patients than a GP, and physiotherapists will probably see a number in between depending where on the patient pathway they sit.

Physiotherapists working at an advanced practice level are likely to see more patients with CES as their patient population is likely to be more complex. Patients seeing advanced practitioners will often have previously failed conservative intervention or may have more serious health problems.

Regardless of the healthcare setting or clinician, it is still important to have a framework, which supports the early identification and management of patients with suspected CES. Timely diagnosis is essential to avoid life-changing outcomes for patients; it is estimated that one fifth of patients will have a poor outcome, with on-going treatment for bladder, bowel and sexual dysfunction, along with significant psychosocial consequences (Gardner et al, 2011).

The best and most widely used model that currently exists to aid in the identification of CES is the system of Red Flags, although these previously considered cornerstones of safe practice have been called into question. Underwood and Buchbinder (2013) suggest that screening for red flags in general is "a popular idea that didn't work and should be removed from guidelines". A number of authors including Henschke \& Maher (2006), Downie et al (2013) and Verhagen et al (2016) have published high quality review papers demonstrating that Red Flags have a weak evidence base. These systematic reviews have focused on the diagnostic accuracy of specific Red Flags. Due to the combined challenges of the relatively rare prevalence of serious pathology in the total back pain population, and the significant difficulty in designing high quality studies in this area, the findings that most Red Flags are not good predictors of serious pathology, is unsurprising. Most recently Cook et al (2017) called for a paradigm shift away from Red Flags stating "...red flag screening is not consistent with best practice in LBP management".

In light of this, it would be fair to question the utility of current red flags for CES and to ask what can we do as treating physiotherapists to manage these patients. To answer this question, it is useful to go back to basics and consider the clinical interaction between a physiotherapist and a patient arriving for their first appointment with a complaint of low back pain, whether it is $4 \mathrm{pm}$ on a Friday or first thing Monday morning. The physiotherapist needs to decide in a limited timeframe whether the patient's problem is suitable for physiotherapy management (keep), or whether the patient needs to be referred to other medical personnel or sent for further investigation (refer) and if so, in what time frame (emergency/soon/routine).

These are vital decisions to make, as CES can have permanent life changing consequences if not acted upon in a timely manner; in the UK the current guidance is that patients should be sent for an emergency MRI and surgical opinion on the same day (Germon et al, 2015). The physiotherapist has a professional duty of care and a legal responsibility to provide appropriate and timely care. In cases of suspected CES this duty is clearly to refer rather than to keep. 
Current World Confederation for Physical Therapy (WCPT) guidelines describe the keep/refer decision making as a core element of physiotherapy practice around the management of patients with potentially serious medical pathology (WCPT, 2011), and this is clearly the case with CES. So, if the current Red Flags do not withstand scrutiny, what should we use to inform our keep/refer decision with a patient in front of us who may have CES? Clinicians must be able to identify true CES from the many conditions that can masquerade as CES, using sensitive and thorough questioning and examination.

\section{Definition of CES}

In the past there has been little consensus in the literature as to how CES is defined. For example Fraser et al, (2009) reported that were 17 different definitions of CES. However, this is improving and 5 characteristic features of CES are now becoming consistently recognised (Todd and Dickson, 2016)

1. Bilateral neurogenic sciatica - Pain associated with the back and/or unilateral/bilateral leg symptoms may be present.

2. Reduced perineal sensation - Sensation loss in the perineum and saddle region is one of the most commonly reported symptoms.

3. Altered bladder function leading to painless urinary retention - Bladder dysfunction is the other most commonly reported symptom and can range from increased urinary frequency, difficulty in micturition, change in urine stream, urinary incontinence and urinary retention.

4. Loss of anal tone - loss or reduced anal tone may be evident if a patient reports bowel dysfunction. Bowel dysfunction may include faecal incontinence, inability to control bowel motions, inability to feel when the bowel is full with consequent overflow.

5. Loss of sexual function - Sexual dysfunction is not widely mentioned in the literature but is an important aspect of health and wellbeing that needs discussion with patients, despite the potential embarrassment for both patient and therapist.

These features are key in helping to standardise the assessment of these patients and should form the basis of the subjective and physical examination of any patient suspected of having CES.

The British Association of Spinal Surgeons (BASS) has produced a statement which is clinically useful and reflects the above 5 features, stating that "a patient presenting with acute low back pain with a suggestion of a disturbance of their bladder or bowel function and/or saddle sensory disturbance should be suspected of having CES"( Germon et al, 2015).

One of the key issues which has important ramifications for physiotherapy practice, (in particular the initial keep/refer decision), is that early symptoms of CES are often subtle and vague (Bin et al, 2009, Sun et al, 2014). This is compounded by the fact that as the condition progresses, the signs and symptoms do not develop in a recognised pattern (Bin et al,2009). Sun et al (2014) confirm that there is no set chronology of presenting symptoms, creating uncertainty and difficulty with early diagnosis. This is particularly challenging for physiotherapists who often rely on pattern recognition to inform clinical decision making, however knowing that that there is no pattern to look for is paradoxically actually quite useful (Greenhalgh et al, 2015). Todd and Dickson (2016) describe four 
separate stages of CES (Table 1), which are characterised by progressive sensory and motor deficits in lower extremities.

Table 1. Four stages of CES (Todd and Dickson 2016)

\begin{tabular}{l|l}
\hline CESS & - Bilateral radicular pain \\
Suspected & Urinary difficulties of neurogenic origin \\
\hline CESI & $\begin{array}{l}\text { - Altered urinary sensation } \\
\text { - Loss of desire to void } \\
\text { Incomplete }\end{array}$ \\
& - Poor urinary stream \\
& Neurogenic retention of urine \\
\hline CESR & - Painless urinary retention and overflow \\
Retention & incontinence where the bladder is no longer \\
& - under executive control \\
\hline CESC & - Abjective loss of CE function \\
Complete & - Patulous anus (spread open) \\
& - Paralysed insensate bladder and bowel
\end{tabular}

The boundary between these stages is blurred rather than discrete and the probability of a CES patient progressing through the stages e.g. from CESS through to CESR and how quickly this may occur cannot be predicted.

In light of the above definition and guidance, what should we be doing to help us make a clinical decision about whether to keep or refer the patient?

\section{Subjective Examination}

The subjective history is the most important aspect of the examination, particularly early in the presentation of a patient with CES, as the subtle and vague symptoms related to early CES need to be identified using clear and unambiguous methods of communication (Bin et al, 2009, Sun, 2014). However, in the context of severe pain, patients with CES have expressed their difficulty in concentrating on clinical questions, especially when they appear to have no relevance to the pain they are suffering e.g. 'what has bladder function got to do with my severe back pain?' (Greenhalgh 
et al, 2015). Patients with severe pain that is all-encompassing may also struggle to identify early subtle changes in bladder and bowel function and in saddle sensation (Whitaker at al, 2015).

It goes without saying that good communication is a core requisite skill in physiotherapy practice and in the clinical context these skills are honed over time, with the experience of many hours of patient contact (patient 'mileage'). However, what is occasionally overlooked is the patient's need to understand the relevance of the questions they are being asked, and their need to fully appreciate the importance of the questions or of the consequences of the answers given, especially in the presence of severe pain.

Greenhalgh et al (2015) found that one of the key problems in communication was the technical/ medical language used by clinicians. The patient participants in the study emphasised the need for clinicians to use clear and some would say explicit language that could be readily understood during a consultation. For example, male patients understood clearly 'Do you have a change in ability to get an erection or ejaculate?' However, when questioned about 'problems with sexual function' patients all thought that the question related to being sexually active.

The patients went on to suggest that a CES cue card may be helpful for clinicians to use during the subjective examination, firstly to help frame, and then focus attention during this part of the assessment on the important and sensitive CES questions (Figure 1). It could also help clinicians avoid some of the embarrassment associated with asking some of the more sensitive questions. The patients also suggested that the CES cue card should map against a patient credit card (Figure 2), using the same questions that the patient could keep for future reference. The patient credit card contains additional information including timely action to take should symptoms develop. The credit card can also be used by patients to facilitate future clinical communication, particularly in emergency settings, to help express embarrassing yet clinically important symptoms. (This card is available in 30 different languages). A patient in the study reflected,

"If I had gone to the GP and he had given me a card with CES [On]...I could have been probably in A\&E at the weekend rather than wait until Wednesday...so it would have been 2 or 3 days prior that I could have been in" (Gareth) 


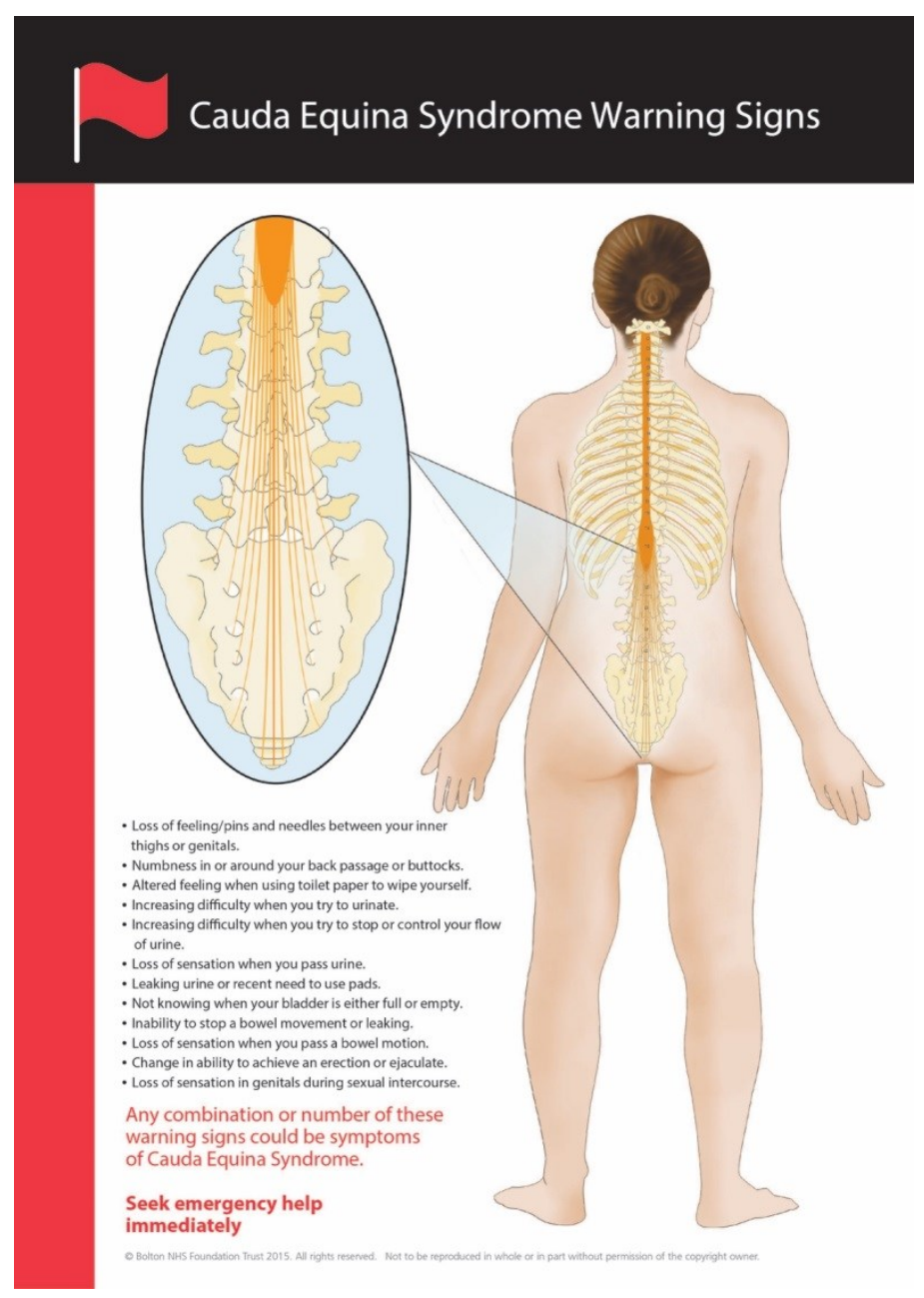

Figure 1 CES Clinical Cue Card reproduced with permission from Sue Greenhalgh and James Selfe

\section{Cauda Equina Syndrome Warning Signs}

- L oss of feeling/pins and needles between your inner thighs or genitals

- Numbness in or around your back passage or buttocks

- Altered feeling when using toilet paper to wipe yourself

- Increasing difficulty when you try to urinate

- Increasing difficulty when you try to stop or control your

flow of urine

- Loss of sensation when you pass urine

- Leaking urine or recent need to use pads

- Not knowing when your bladder is either full or empty

- Inability to stop a bowel movement or leaking

- Loss of sensation when you pass a bowel motion

- Change in ability to achieve an erection or ejaculate

- Loss of sensation in genitals during sexual intercourse

Figure 2 CES Patient Credit Card reproduced with permission from Sue Greenhalgh and James Selfe

During the subjective examination establishing the chronology of the history of the present condition in detail is key as timing is of paramount importance in the successful management of CES. The onset of back and or leg pain is significant but precisely when symptoms relating to the parasympathetic nerve supply began is critical. As stated earlier there is no way of predicting who 
will progress from Cauda Equina Suspected (CESS) to Cauda Equina in Retention (CESR), or how quickly this may happen (Table 1), so the importance of precise recording of the timing of chronology cannot be overestimated. Clinicians need to clearly establish if symptoms are better, worse or the same. Improving pain does not necessarily mean the condition is improving. Improved neurological status must be confirmed before this can be assumed. Establishing the pattern of pain through 24 hours and recording the precise area of pain, pins and needles and numbness is essential. Establish if these symptoms have been experienced before or if they are new or different in some way? Further questioning should include the following;

- What treatments have been tried (including medication), is helpful on a number of levels. Many medications cause symptoms that masquerade as CES (Woods e al, 2015). This does not mean that symptoms can be ignored and attributed to drugs; however, medication could be contributing to the bladder, bowel and sexual dysfunction (Table 2). Similarly, pain can cause bladder retention.

- What is the past medical history status? Previous diagnosis of disc pathology or spinal stenosis may be significant. Previous history of serious conditions such as cancer must be noted and may be important. Similarly many co-morbidities could masquerade as CES e.g. Diabetes, Multiple Sclerosis, Benign prostatic hyperplasia, (Woods et al, 2015).

- Has there been any recent or past spinal surgery and/or any history of osteoporosis? Cauda Equina Compression is not solely caused by discal compression but could be from a mass caused by infection or haematoma. Similarly, a fragment of bone from a retro-pulsed vertebral insufficiency fracture could cause CES. Medication comorbidities are discussed later.

Once the subjective history gives a suggestion of CES a full physical examination should be undertaken.

\section{Physical Examination}

A full neurological assessment should be performed to establish if any dermatomal sensory loss, myotomal weakness or reflex change is present. If there are any suspicions of a higher lesion or central nervous system lesion causing symptoms, then upper motor neurone tests such as the plantar (Babinski) response, clonus testing, muscle tone testing, joint position sense and gait should also be included in the examination.

A digital rectal examination (DRE) is currently considered in UK guidelines as essential to evaluate loss of anal sphincter tone (Todd \& Dickson, 2016). Whilst this is an established test for patients with suspected CES, the evidence for its use is weak, and there is now some debate as to whether it should be included in the examination at all. The evidence suggests that there is no direct correlation between a finding of decreased anal tone and the presence of cauda equina compression on MRI scan (Gooding et al 2012) and that the physical tests have low sensitivity and specificity (Balasubramanian et al 2010: Bell et al 2007). The reliability of the test has also been shown to be variable, as has the written documentation of findings of the examination (Tudose et al 2017). Any clinician opting to carry out a DRE must be appropriately trained and competent in the procedure; it is also mandatory that a chaperone is offered to the patient during this sensitive examination.

Current evidence suggests that the presence of sensory disturbance in the saddle region is a more valid and reliable indicator of CES (Gitelman et al 2017;). Sensation to light touch and pin prick 
throughout the saddle region including buttocks, inner thighs and perianal region must also be tested (Todd and Dickson, 2016). Like DRE testing, these 'intimate' tests must only be performed by an appropriately trained clinician with a chaperone for the benefit of both the patient and the clinician.

The ultrasound assessment of residual bladder volume post voiding is commonly used in the emergency department to help test for CES. Again, whilst this is widely used, the evidence to support its validity in assessment for CES is not well established, as authors have suggested a variety of residual volumes (100/200/500ml) may be important (Domen et al 2009; Angus et al 2015; Venkatesan et al 2015).

We recognise that there is ongoing discussion and a current lack of consensus about which specific physical tests should be used to help with the diagnosis of CES, especially DRE. However, guidelines recommend that a full neurological examination including saddle sensory testing for light touch and pin-prick, and anal tone testing is undertaken in the presence of a chaperone by clinicians who are trained and competent to do so. Failure to undertake this battery of tests and gain as complete a clinical picture as possible may leave the clinician open to challenge and potential litigation.

\section{CES Masqueraders}

As Germon et al (2015) point out, in the population of patients investigated with MRI to confirm or negate CES a significant number will not have CES. There are many causes for symptoms that masquerade as CES adding to the challenge of accurate and timely diagnosis, this includes comorbidities and perhaps less well known the effects and side effects of some prescription medication, which may be responsible for masquerading symptoms. This includes a number of drugs not prescribed for pain relief. Cholinergic and Anticholinergic drugs influence the parasympathetic nervous system with cholinergic drugs mirroring parasympathetic nervous activity, anticholinergic drugs doing the opposite. Cholinergic drugs (which may be used for glaucoma or myasthenia gravis) therefore can lead to voiding of urine and anticholinergic drugs (used for COPD, urinary incontinence) to the retention of urine (Mtui et al, 2016). In fact, most of the medication used for pain relief in patients with back pain and leg pain can also cause symptoms that masquerade as CES (Table 2). 
Table 2 Analgesic medication used in patients with back pain and leg pain and possible CES symptoms

\begin{tabular}{|l|l|l|}
\hline Prescribed medication type & Example & Possible CES symptoms \\
\hline Opioid Salts & e.g. Tramadol, codeine & $\begin{array}{l}\text { Constipation, reduced gastric } \\
\text { motility, reduced bladder } \\
\text { sensation }\end{array}$ \\
\hline Anticonvulsants & e.g. Gabapentin, Pregabalin & Urinary incontinence \\
\hline Antidepressants & $\begin{array}{l}\text { e.g. Amitriptyline, } \\
\text { Nortriptyline }\end{array}$ & $\begin{array}{l}\text { Retention, sexual dysfunction, } \\
\text { reduced awareness of need to } \\
\text { pass urine }\end{array}$ \\
\hline NSAIDS & e.g. Naproxen, Ibuprofen & $\begin{array}{l}\text { Retention twice as likely in } \\
\text { men than women }\end{array}$ \\
\hline
\end{tabular}

Even decongestants, over the counter cold remedies and recreational drugs can affect bladder function. In relation to comorbidities, benign prostate hyperplasia is the most common cause of retention in men.

Given the large number of potential causes of symptoms masquerading as CES, the clinician must be vigilant, thorough and considered in their approach, and must always, as directed by current guidelines, act immediately if CES is suspected as the cause of the symptoms (Germon et al 2015). Whilst this 'safety first' approach is likely to contribute to the high number of negative MRI scans in people presenting with CES-like symptoms, (a 10\% finding of CES on MRI in those with signs and symptoms is not unusual) the clinician is bound by their duty of care to the patient to exclude CES as a first priority.

\section{CES and litigation}

According to the NHS litigation authority 293 claims were made by CES sufferers between 20102015 , at a cost of 25 million pounds. In $70 \%$ of these cases, patients were aged between 31-50 years (NHSLA 2016).

Consequently, the impact on Physiotherapy in the UK has in recent years become problematic due to increasing numbers of cases of litigation involving Physiotherapists. For those involved in litigation, be that the patient or the clinician, it can be a very stressful and arduous process. It is essential that the clinician protects themselves and their patient by maintaining full and accurate records.

As well as thorough and accurate clinical documentation, it is vital that there are clear pathways and protocols in place to help manage patients with suspected CES. A robust pathway that has been agreed by all members of the multidisciplinary team, that has been widely circulated and made available to all, will help everyone involved in a service manage these patients. Whilst templates are 
available for this, they need to be locally agreed to fit in with how services are configured in different regions and in different countries.

A useful clinical update and accompanying video commissioned by the Chartered Society of Physiotherapy in the UK have recently been published which gives good advice on how to manage patients with suspected CES. These are available at http://www.csp.org.uk/frontline/article/clinicalupdate-cauda-equina-syndrome and the video is available at;

https://www.youtube.com/watch?v=8rRq5QqoK3o

\section{Summary}

CES is rare but can have life changing consequences if not acted upon immediately when suspected. Physiotherapists can play an important role in bringing the patient and surgical team together in a timely manner. If surgical intervention is delayed, irreversible damage can occur to the bladder, bowel and sexual function with ongoing chronic pain. Relevant symptoms which can be a precursor to CES are unilateral or bilateral radicular pain and/or dermatomal reduced sensation and/or myotomal weakness; if symptoms progress with any suggestion of changes in bladder or bowel function however minor, CES should be suspected (Germon et al, 2015). Emergency MRI must be carried out on the same day to confirm or negate CES. The British Association of Spinal Surgeons (BASS) state that, 'Nothing is to be gained by delaying surgery and potentially much to be lost'; surgery should be carried out as soon as is practically possible.' (Germon et al, 2015, Todd and Dickson, 2016).

Clearly not all patients with back pain will develop CES, consequently it is not necessary to warn all patients. Patients who you suspect may have the potential to progress into CES, should be 'safety netted' with watchful waiting, rather than immediately referred (Underwood and Buchbinder 2013, Cook 2017). Warning patients at risk is key to early diagnosis; providing these at-risk patients with appropriate information of what to do should CES symptoms develop can expedite a timely surgical opinion.

Many patients interviewed in the Greenhalgh et al (2015) study ignored significant symptoms such as saddle numbness, placing more weight on their pain levels. Some thought that saddle numbness was the beginning of the recovery process. Patients need to understand the relevance of their symptoms and of the questions being asked as they may not fully appreciate the importance and subsequent consequences if not explained properly.

It is also important that a patient's signs and symptoms are fully documented including the timing of examination findings so that there is a clear record of the patient's journey. There are no predictive signs or symptoms of who will progress through to what stage of CES, hence the value of accurate and timed/dated documentation. This paper outlines the complexity of managing patients with CES. Whilst the evidence continues to evolve, and will continue to produce more robust Red Flags in the future, the current expected best practice standards of care for this patient group is outlined.

It is hoped that in the near future, a new clinical framework for the management of CES and other spinal red flags, including metastatic disease, infection and fractures, will be produced by an 
international working group supported by International Federation of Orthopaedic and Manipulative Physical Therapists (IFOMPT) and the Chartered Society of Physiotherapists (CSP).

\section{Acknowledgements}

Chartered Society of Physiotherapy for funding the Cauda Equina video and to the Physiotherapy Research Fund ; PRF Reference Number; PRF 11/06 for their support.

\section{References}

Angus M, Elmajee M, Verma R, Mohammad S, Siddique I, 2016. The utilisation of post-micturution bladder scan in the assessment with suspected cauda equine syndrome (ces). Posters/The Spine Journal S72-S93

Balasubramanian K, Kalsi P, Greenough C, Kuskoor Seetharam M. 2010. Reliability of clinical assessment in diagnosing cauda equina syndrome. Br J Neurosurg. 24 (4): 383-6

Bell DA, Collie D, Statham PF (2007) Cauda equina syndrome: what is the correlation between clinical assessment and MRI scanning? Br J Neurosurg. 21: 201-3

Bin, M. Hong, W. Lian-shun, J. Wen, J. Guo-dong, S. Jian-gang, S. 2009: Cauda equina syndrome: A review of clinical progress. Chin Med J. 122:1214-22

Chartered Society of Physiotherapy, 2012, Information paper, Pelvic floor examination CSP expectations

Cook C, George S, Reiman M. 2017. Red flag screening for low back pain: nothing to see here, move along: a narrative review. British Journal of Sports Medicine. Doi:10.1136/bjsports-2017-098352

Domen P, Hofman P, van Santbrink H, Weber W. 2009.Predictive value of clinical characteristics in patiets with suspected cauda equine syndrome. European Journal of Neurology. 16: 416-419

Downie A, Williams C, Henschke N, Hancock M, Ostelo R, de Vet H, et al. Red flags to screen for malignancy and fracture in patients with low back pain: Systematic review. BMJ. 2013;347:7095

Fairbank J (2014) Cauda Equina Syndrome - risk management. Journal of Trauma and Orthopaedics: 2 (3): $49-50$

Fraser S, Roberts L, Murphy, E, 2009 Cauda equine syndrome;a literature review of its definition and clinical presentation. Arch Phys Med Rehabil 11;1964-8 
Fuso F, Dias A, Letaif, Cristante A, Marcon R, Pessoa de Barros T, 2013. Epidemiological study of cauda equina syndrome. OActa Ortop Bras. ; 21(3): 159-162.

.Gardner A, Gardener E, Morley T. 2011. Cauda equina syndrome; a review of the current clinical and medical legal position. Eur Spine: 20(5):690-697

Germon T, Ahuja,S, Casey A, Rai A. British Association of Spine Surgeons standards of care for cauda equina syndrome. The Spine Journal 201515 (3), pS2-S4

Gitleman A, Hishmeh, S, Moreli B, Joseph S, Casden A, Kulflik P, Neuwirth M, Stephen M. 2008 Cauda Equina Syndrome; A comprehensive Review. The American Journal of Orthopaedics; 37(11); 556-562

Greenhalgh S, Truman C, Webster V, Selfe J (2015) An Investigation into the Patient Experience of Cauda Equina Syndrome (CES). Physiotherapy Practice and Research, 36: 23-31

Gooding B, Higgins M, Calthorpe D. 2013 Does rectal examination have any value in the clinical diagnosis of cauda equina syndrome? British Journal of Neurosurgery;27 (2): 156-159

Health and Care Professions Council, 2013, Standards of proficiency - Physiotherapists

Henschke N, Maher C Refshauge K, Herbert R, Cumming R, Bleasel J, York J, Das A, McAuley J. 2006. Prognosis of acute low back pain; design of a prospective inception cohort study. BMC Musculoskeletal Disorders. 7:54

Langridge N. Roberts L, Pope C. 2015, The role of clinician emotion in clinical reasoning; balancing the analytical process. Manual Therapy doi 10.1016/j.math. 2015.06.007

Mtui E. 2016. Fitzgerald's Clinical Neuroanatomy and Neuroscience,7th Edition. Elsevier, London NHS Litigation Authority 2016 Cauda Equina Syndrome. www.nhsla.nhs.uk \{accessed 29/01/18\}

Sun JC, Xu T, Chen KF, Qian W, Liu K, Shi JG, Yuan W, Jia LS. Assessment of Cauda Equina Syndrome progression pattern to improve diagnosis. Spine. 2014; 39 (7): 596-602. 34.

Todd, N V; Dickson, R A . Standards of care in cauda equina syndrome. British Journal of Neurosurgery. 2016, 30 (5):518-522.

Tudose A, Hogg F, Giamouriadis A, Low D, Bassi S, Selway R. 2017. FP1-7: The neurosurgical rectal examination (PR) -obsolete practice or standard of care? Proceedings of the 2017 Spring Meeting of the Society of British Neurological Surgeons British Journal of Neurosurgery. 31 (2):119-158

Underwood M, Buchbinder R. Red flags for back pain. BMJ 2013;347:f7432

Venkatesan M, Nasto L, Grevitt M, Tsegaye M. Is post-void bladder scan a useful adjunct to the clinical examination for prediction of cauda equine syndrome? 2017. BASS abstracts/The Spine Journal S3-S22

Verhagen AP1, Downie A2,3, Popal N4, Maher C2, Koes B. 2016. Red flags presented in current low back pain guidelines: a review. Eur Spine J. 25(9):2788-802. 
Whitaker K, Scott S, Wardle J. 2015 Applying symptom appraisal models to understand sociodemographic differences in response to cancer symptoms; a research agenda

Woods E, Greenhalgh S, Selfe J (2015) Cauda Equina Syndrome and the challenge of diagnosis for physiotherapists: a review Physiotherapy Practice and Research.36:81-86

World Confederation for Physical Therapy. 2011.WCPT guideline for physical therapist professional entry level education. London, UK:WCPT; Available from: http://www.wcpt.org/guidelines/entrylevel-education 